\title{
Value of Cytokeratin-18 as a non-invasive diagnostic biomarker of non- alcoholic steatohepatitis (NASH)
}

Alaa Habib, Mahmoud Awad, Dalia Shaheen, Somaia Shehab- Eldeen, Sheren Younes, Esmat Mohammed

\begin{abstract}
Summary
Background and study aim: Liver biopsy couldn't be utilized for screening of nonalcoholic steatohepatitis (NASH), as it is considered invasive, and has many complications. The study aims to investigate the value of serum levels of CK -18 fragments as a diagnostic biomarker for NASH and NAFLD and its correlation with severity of $\mathrm{NASH}$ as measured by NAFLD activity score (NAS) of liver biopsies. Patients and methods: A total number of 46 subjects with biopsy-proven non-alcoholic steatohepatitis (NASH group) and 54 subjects with borderline $\mathrm{NASH}$, simple steatosis and normal liver tissue (non-NASH group) as well as 30 age-matched healthy volunteers were included in the study. Scoring of liver biopsies using the NAFLD activity score (NAS) and measurement of $\mathrm{CK}-18$ in sera was done. Results: The serum level of cytokeratin-18 was significantly higher in the NASH group when compared to non-NASH group $(P=0.0123)$ or controls $(P=0.00001)$. Using the ROC curve, the optimal value of cytokeratin-18 was $487 \mathrm{U} / \mathrm{L}$, with sensitivity $69 \%$ and specificity $84.5 \%$ in detecting NASH. Serum CK-18 levels were significantly correlated to the disease severity as measured by liver biopsy (degree of steatosis, fibrosis, lobular inflammation, and ballooning) in NASH patients. Conclusions: Serum CK18 could be used as a non-invasive diagnostic serum marker for patients of NAFLD and NASH.
\end{abstract}

\section{Keywords: Cytokeratin-18, NASH, NAFLD}

\author{
Medical Journal of Viral Hepatitis \\ (MJVH) 2019; 4 (2) - pp. 65-74
}

Received: $3 / 11 / 2018$

Revised: 7/2/2019

Accepted: 29/3/2019

Published Online: 25/4/2019

(Alaa Habib, Mahmoud Awad) Internal Medicine dept, Faculty of Medicine, Mansoura Univ., Egypt.

(Dalia Shaheen) Medical Biochemistry dept., Faculty of Medicine, Mansoura Univ., Egypt. (Somaia Shehab-Eldeen) Tropical Medicine dept., Faculty of Medicine, Menoufia Univ., Egypt.

(Sheren Younes) Pathology dept., Faculty of Medicine, Menoufia Univ., Egypt.; Histopathology dept., College of Medicine, Princess Nourah Bint Abdul Rahman Univ., KSA (Esmat Mohammed) Radiology dept., National Institute of Cancer, Cairo University, Egypt

\footnotetext{
* CA: Alaa Habib

alaahabib75@hotmail.com
}

\section{Introduction}

Nonalcoholic fatty liver disease (NAFLD) is a common and prevalent chronic liver disease worldwide with prevalence ranges from $15 \%$ to $30 \%^{1,2}$. This prevalence appears to be increasing daily ${ }^{3}$. There is a solid relationship between NAFLD and metabolic syndrome (obesity, insulin resistance, Type 2 diabetes mellitus, hyperlipidemia, and hypertension) $)^{4,5}$. Two continuous yet distinct categories of NAFLD exist, which are nonalcoholic fatty liver (NAFL) and nonalcoholic steatohepatitis (NASH), but in contrast, NASH typically has a progressive nature. Almost $25 \%$ of affected patients develop liver cirrhosis which is further complicated with portal hypertension and even hepatocellular carcinoma ${ }^{6}$. The diagnosis of NAFLD depends on the elevation of liver enzymes, the diagnostic ultraso- und and pathologic findings of steatosis ${ }^{1}$. Liver biopsy is considered an effective definitive diagnostic tool for NASH, that assists making the diagnosis and provides predictable factors for disease progression, yet it is still of limited use as a screening tool being invasive, and carries the risk of hemorrhage ${ }^{7}$. Non-invasive biomarker tests for fibrosis or steatosis have been developed. It is calculated using the results of other laboratory tests and it is correlated to the degree of fibrosis like Fibro Tests (5), or steatosis like Steato Test $(\mathrm{ST})^{\mathbf{8}}$. The balance between cell proliferation and apoptosis on the other side is believed to keep and maintain tissue homeostasis. In liver injury conditions as in NAFLD, the progression of the disease is thought to arise from hepatocyte apoptosis ${ }^{9}$. It is known that the 
deregulation of tissue homeostasis is likely a cause of several liver diseases. Liver diseases like viral hepatitis, alcoholic hepatitis, NAFLD, liver cirrhosis, cholestatic liver disease, and hepatocellular carcinoma are believed to be related to apoptosis in their development and progress$\operatorname{sion}^{10}$. Both intrinsic and extrinsic pathways of apoptosis are believed to be involved in NASH pathogenesis $^{11}$. Cytokeratin-18 (CK-18) is an intracellular intermediate filament protein. It is abundantly expressed in the liver. It is cleaved in apoptosis and has been implicated in various liver diseases ${ }^{12}$. Many clinical cohorts documented that, in obese patients or patients with insulin resistance, high serum levels of CK-18 fragments were associated with hepatocyte inflammation, necrosis, and fibrosis. Taking the above facts in consideration, $\mathrm{CK}$ - 18 could be considered as a potential non-invasive indicator to predict the hepatic histological manifestation of NASH ${ }^{1,13-16}$. The current study aims to address the diagnostic role of serum levels of CK-18 fragments in NASH and address its potential value for differentiating NAFL from NASH and the relation between its serum levels and pathologic criteria of NASH.

\section{Subjects \& Methods}

This study is a cross-sectional uni-central observational study that was done on a total number of 100 patients, spanning the period between March to December 2017. Cases with a suspected diagnosis of NASH were selected from outpatient clinics of internal medicine department of Riyadh Care Hospital, KSA. Thirty age and sex-matched unaffected individuals were used as a control group.

\section{Inclusion criteria}

They included Patients above 18 years, diagnosed with NASH and NAFLD for whom the diagnosis was confirmed by laboratory tests, ultrasonography, and liver biopsy. Patients from both genders were eligible for the study, and written informed consent was signed by each case. The protocol of the study was reviewed and accepted by the local ethics committee of the hospital. It follows the provisions of the World Medical Association (Declaration of Helsinki) for experiments in humans.

\section{Exclusion criteria}

They included patients with any liver disease due to any other cause like autoimmune hepatitis, Wilson's disease, chronic viral hepatitis, hemochromatosis, $\alpha 1$-antitrypsin deficiency, primary sclerosing cholangitis and primary biliary cir- rhosis, toxins, and medication-induced liver steatosis or hepatotoxicity, and hepatocellular carcinoma. Also, patients with alcoholic fatty liver disease as defined by consumption of alcohol $\geq 40 \mathrm{~g} /$ day for males or $\geq 20 \mathrm{~g} /$ day for females in the past five years or excess alcohol consumption of $\geq 140 \mathrm{~g} /$ week for males or $\geq 70 \mathrm{~g} /$ week for females were excluded as well. None of the patients was pregnant or having evidence of thyroid dysfunctions, type I diabetes or biliary obstruction. All enrolled cases were subjected to detailed history taking, physical examination and investigations including abdominal ultra-sonography, laboratory investigations, and ultr-asonography-guided liver biopsy (except for the control group). Abdominal ultrasonography was done to all individuals by the same operator and steatosis was being graded into 0: absent, 1: mild, 2: Moderate and 3: severe. Liver biopsies were performed by an expert interventional radiologist that sent the samples to an expert pathologist for assessment of the histopathological changes of NASH and excluding other chronic liver diseases in all individuals except the control group. According to NIDDK (National Institutes of Diabetes, Digestive, and Kidney Disease) NASH Clinical Research Network Scoring System ${ }^{17}$, our patients with suspected NAFLD can be classified according to their liver histology into four groups: definitive NASH, borderline NASH, simple fatty liver and normal liver tissue. In a secondary analysis, all individuals were subdivided into 3 groups: Group A: includes patients with definitive NASH or NASH group. Group B: includes patients with borderline NASH, simple fatty liver or normal liver tissue. Collectively named non-NASH group. Group C: This included individuals of matched age and sex, of no history of abnormal liver biochemical tests or ultrasonography and currently with normal liver biochemical tests and ultrasonography as a control group.

\section{Clinical data}

Collected clinical data included age, gender, height, weight, body mass index (BMI), smoking (as never smoked $=0$, ex- smoker $=1$, recent smoker $=2$ ).

\section{Laboratory assessment}

Blood samples were drawn from patients then stored at $-80{ }^{\circ} \mathrm{C}$. This was done at the same time with the liver biopsy. The laboratory assessment for all patients included: 1- Liver function 
tests including serum aspartate transferase (AST), serum alanine transferase $(\mathrm{ALT})^{\mathbf{1 8}}$, alkaline phosphatase (ALP) ${ }^{\mathbf{1 9}}$, gamma-glutamyl transferase $(\mathrm{GGT})^{\mathbf{2 0}}$, and bilirubin ${ }^{21}$, and serum albumin. 2- Viral B \& $C$ hepatitis screen via HBs $\mathrm{Ag} \&$ Anti- HCV antibody was assayed by ELISA. 3- Renal function tests including creatinine and uric acid (UA) were assessed using Reflotron plus. 4- Measurements of total, HDL cholesterol, and triglycerides ${ }^{22}$. 5- Quantitative measurement of C-reactive protein $(\mathrm{CRP})^{\mathbf{2 3}}$. 6- International normalized ratio (INR), platelets, white blood cell (WBC) and hemoglobin were assessed. 7- Anti-nuclear (ANA), anti-smooth muscle (ASMA) and anti-liver/kidney microsomal (LKMA) antibodies were assayed by ELISA. 8- Quantitative measurement of ferritin was done using an immunoturbidimetric. assay (Kamiya Biomedical Company, Seattle, WA). 9- Alpha-1 antitrypsin concentrations were determined using immunoturbidimetric assay kits (DiaSorinInc, Stillwater, MN). 10- Ceruloplasmin was detected colorimetrically. 11- Diagnosis of diabetes mellitus (DM) was depending on a history of hypoglycemic medications and/or fasting glucose (FG) of $126 \mathrm{mg} \mathrm{dL}-1$ or more and diagnosis of hypertension was based on a history of blood pressure medications or twice/ day $\mathrm{BP}$ at $\geq 140 / 90 \mathrm{mmHg}$. Dyslipidemia was assessed by medications history and/or fasting total cholesterol $>200 \mathrm{mg} \mathrm{dL}-1$ and/or fasting TG > $150 \mathrm{mg} \mathrm{dL}-1$.

\section{Cytokeratin-18 (CK-18) Fragments (M30} ANTIGEN)

Sera for CK18 fragments of the patients and the healthy control group were assessed in duplicate for CK18-Asp196 by means of the M30-Apoptosense ELISA (PEVIVA AB, Bromma, Sweden), (M30 Apoptosense $\AA$ ELISA (PEVIVA $®)$ ).

\section{Histopathology}

Liver biopsies of the patients were obtained under moderate sedation by a 16- gauge Klatskin needle. A $2.5 \mathrm{~cm}$ is the minimal length of the specimen. Fixation of the biopsies was done by $10 \%$ neutral buffered formalin solution. Then the sections were concurrently stained with Hematoxylin-Eosin and Masson's trichrome. Histopathological examination was done by an experienced pathologist and scoring of the liver biopsies was done according to the NAFLD activity score (NAS) in which: steatosis score from 0 to 3 , lobular inflamemation score from 0 to 3 , ballooning score from 0 to 2 and fibrosis was scored from 0 to 4 , fig $_{\text {s }}(1 \& 2)^{17}$. The histological NASH was defined as the sum of all these scores (steatosis, lobular inflammation, cell ballooning, and fibrosis). Patients with nonalc-oholic fatty liver (NAFLD) have NAS score of $<3$, patients with borderline nonalcoholic steatohepatitis (NASH) have a score of 3-4, and patients with NASH have a score of $\geq 5$ corresponds ${ }^{17}$.

\section{Statistical Analysis}

It was done using the SPSS (Statistical Package for the Social Sciences) software version 20.0 for Windows (SPSS, Inc., Chicago, IL). Data were expressed as median $\left(25^{\text {th }} \& 75^{\text {th }}\right.$ centiles for non-normally distributed data) and as mean \pm SD for normally distributed variables. The $\chi^{2}$ test used to compare proportions and categorical variables. Normality of distribution of continuous variables was tested using a Kolmogorov-Smirnov test. Mann-Whitney test and Kruskal-Wallis tests were used in group comparisons for non-normally distributed variables. Analysis of variance (ANOVA) and unpaired Student's $t$-test was used for normally distributed variables.

\section{Results}

The clinical and laboratory parameters of the pati-ents (NASH and non-NASH) and the control group are displayed in tab. (1). Our current study included 54 patients with nonNASH with mean age $46.7 \pm 13.6$ years, 31 were males and 23 were females, with BMI of $36.23 \pm 5.9 \mathrm{~kg} / \mathrm{m}^{2}$. The NASH group included 46 patients, 27 were males and 19 were females, with mean age $57.9 \pm 15.3$ years with BMI of $39.76 \pm 6.9 \mathrm{~kg} / \mathrm{m}^{2}$. The control group included 30 volunteer individuals, 19 males and 11 females, with mean age $55.65 \pm 9.52$ years and BMI was $55.65 \pm 9.52 \mathrm{~kg} / \mathrm{m}^{2}$. Of the 54 non-NASH patients, $14(25.6 \%)$ patients had type 2 diabetes mellitus, 13 (24\%) patients had hypertension and $34(62.9 \%)$ patients had dyslipidemia. Of the 46 NASH patients, 13 (28.3\%) patients had type 2 diabetes, $12(26.1 \%)$ had hypertension and $30(65.2 \%)$ patients had dyslipidemia. Of the control group, 3 patients (10\%) had diabetes, 7 pati-ents (23.3. \%) had hypertension and 10 patients (33.3\%) had dyslipidemia. There were significant statistical differences in the prevalence of diabetes, dyslipidemia, and obesity among non-NASH and NASH groups compared to the control group. There were significant statistical differ-ences in AST, ALT, GGT, FBG, TC, TG, HDL, BMI 
and of HOMA-IR of both NASH and nonNASH groups compared to the control group. AST, Gamma-GT, HOMA- IR, ferritin and hcCRP showed statistically significant differences between NASH and non-NASH groups. Ferritin and HDL showed statistically significant differences between NASH and control groups. Cytokeratin-18 (Ck-18) In studied groups Table (2) shows serum CK-18 levels in the patients (NASH and non-NASH) and the control group. The mean CK-18 of the NASH group was $754 \pm 467.4 \mathrm{U} / \mathrm{L}$, the non-NASH group was $443 \pm 267.7 \mathrm{U} / \mathrm{L}$, and the control group was $154 \pm 67.3$ U/L. Serum CK-18 was statistically significant higher in non-NASH and NASH groups in relation to the control group ( $\mathrm{P}$ values were 0.0001 and 0.00001 respectively). Moreover, it was statistically significant higher in the NASH group in relation to the non-NASH group ( $\mathrm{P}$ value was 0.0123 ).

\section{Receiver Operating Characteristic (Roc) Curve}

Figure (3) showed ROC curve showing the diagnostic sensitivity and specificity of CK-18 in diagnosing NASH. ROC curve was performed to have a cut-off value of CK-18 and to detect the predictive discriminative value of CK-18 in NASH diagnosis. At the optimal value of 487U/L, the area under the curve (AUC) of CK-18 was 0.81 with sensitivity $69 \%$ and specificity of $84.5 \%$ in detecting NASH. The positive predictive value (PPV) and negative predictive value (NPV) at the cut-off of $487 \mathrm{U} / \mathrm{L}$ for CK-18 for the diagnosis of NASH were $83 \%$ and $71 \%$ respectively. The relationships between CK-18 and clinical and laboratory and histopathologic parameters of the NASH patients: Table (3) showed the correlations of CK-18 to the clinical and laboratory parameters of the NASH patients. Multiple regression analyses were done and resulted in significant positive associations between serum CK-18 and BMI $(r=0.71, p<0.001)$, FBG $(r=0.312, p=0.017)$, HOMA-IR $(r=0.179, \mathrm{p}=0.021)$, hc-CRP $(\mathrm{r}=0.64$, $\mathrm{p}<0.001)$, degree of steatosis $(\mathrm{r}=0.421, \mathrm{p}<0.001)$, degree of fibrosis $(r=0.74, p<0.001)$, lobular inflammation $(\mathrm{r}=0.56, \mathrm{p}<0.001)$ and ballooning $(\mathrm{r}=0.39, \mathrm{p}<0.001)$ in our NASH patients.

Table (1) Clinical and laboratory parameters of the patients (NASH and non-NASH) and the control group

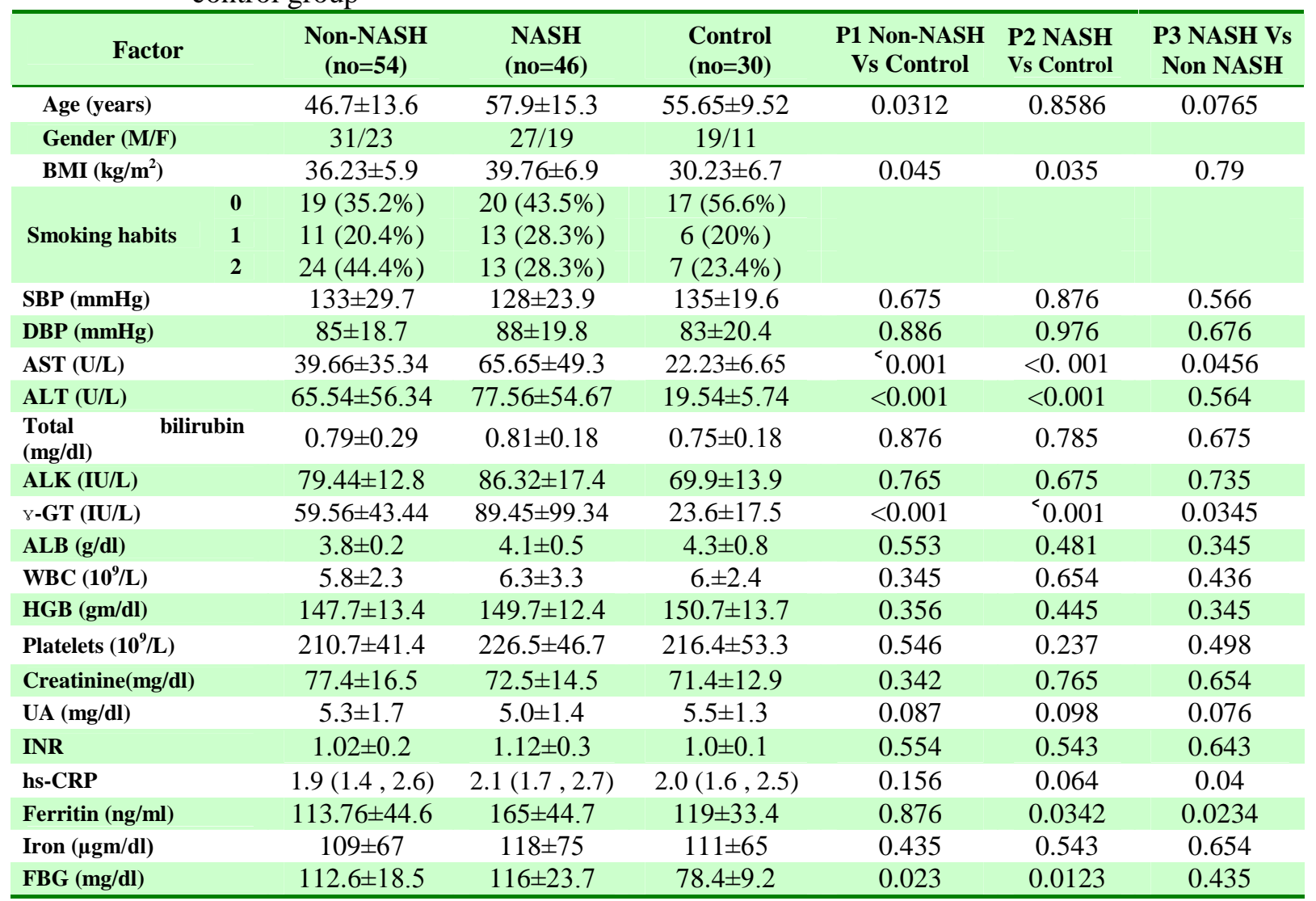




\begin{tabular}{lcccccc}
\hline TC $(\mathbf{m g} / \mathbf{d l})$ & $197 \pm 35.9$ & $207 \pm 39.9$ & $157 \pm 15.9$ & 0.0345 & 0.0234 & 0.654 \\
TG $(\mathbf{m g} / \mathbf{d l})$ & $157 \pm 75.9$ & $167 \pm 85.6$ & $127 \pm 35.9$ & 0.0453 & 0.0332 & 0.564 \\
HDL $(\mathbf{m g} / \mathbf{d l})$ & $47.6 \pm 18.5$ & $53.6 \pm 23.5$ & $69.6 \pm 18.5$ & 0.0132 & 0.0386 & 0.931 \\
HOMA-IR & $3.1 \pm 0.87$ & $4.3 \pm 0.37$ & $1.5 \pm 0.07$ & 0.003 & 0.0001 & 0.0321 \\
DM & $14(25.6 \%)$ & $13(28.3 \%)$ & $3(10 \%)$ & 0.026 & 0.014 & 0.651 \\
HTN & $13(24 \%)$ & $12(26.1 \%)$ & $7(23.3 \%)$ & 0.764 & 0.546 & 0.654 \\
\hline DLP & $34(62.9 \%)$ & $30(65.2 \%)$ & $10(33.3 \%)$ & 0.002 & 0.001 & 0.231 \\
\hline
\end{tabular}

BMI: body mass index, SBP: systolic blood pressure, DBP: diastolic blood pressure, $\boldsymbol{A S T}$ : aspartate transferase, ALT: alanine transferase, ALK: alkaline phosphatase, Gamma-GT: gamma glutamyl transpeptidase, ALB: albumin, WBC: white blood cells, HGB: haemoglobin, $\boldsymbol{U A}$ : uric acid, INR: international normalization ratio, hs-CRP: high-sensitivity $C$-reactive protein, $\boldsymbol{F B G}$ : fasting blood glucose, $\boldsymbol{T C}$ : total cholesterol, $\boldsymbol{T G}$ : triglycerides, HDL: high density lipoprotein, HOMA-IR: Homeostatic Model Assessment of Insulin Resistance, DM: diabetes mellitus, HTN: hypertension, DLP: dyslipidemia.

Table (2) Serum CK-18 levels in patients (NASH and non-NASH) and the control group

\begin{tabular}{ccccccc}
\hline Factor & $\begin{array}{c}\text { Non-NASH } \\
(\mathrm{no=54})\end{array}$ & $\begin{array}{c}\text { NASH } \\
(\mathrm{no}=\mathbf{4 6})\end{array}$ & $\begin{array}{c}\text { CONTRO } \\
\text { L }(\mathrm{no}=\mathbf{3 0})\end{array}$ & $\begin{array}{c}\text { Pon- NASH VS } \\
\text { CONTROL }\end{array}$ & $\begin{array}{c}\text { P2 } \\
\text { NASH VS } \\
\text { CONTROL }\end{array}$ & $\begin{array}{c}\text { P3 } \\
\text { Non- NASH } \\
\text { VS NASH }\end{array}$ \\
\hline CK-18 (U/L) & $\mathbf{4 4 3} \pm \mathbf{2 6 7 . 7}$ & $\mathbf{7 5 4} \pm \mathbf{4 6 7 . 4}$ & $\mathbf{1 5 4} \pm \mathbf{6 7 . 3}$ & $\mathbf{0 . 0 0 0 1}$ & $\mathbf{0 . 0 0 0 0 1}$ & $\mathbf{0 . 0 1 2 3}$ \\
\hline
\end{tabular}

CK-18: cytokeratin 18

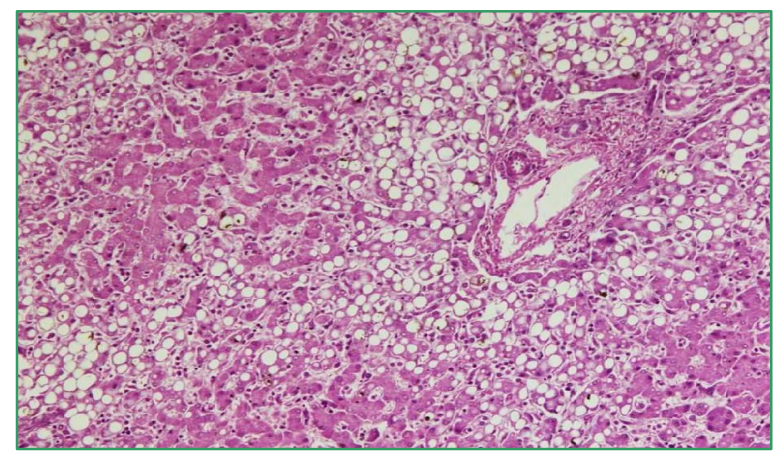

Figure (2) Liver biopsy from NAFLD patient shows extensive periportal steatosis (Original magnification X200)
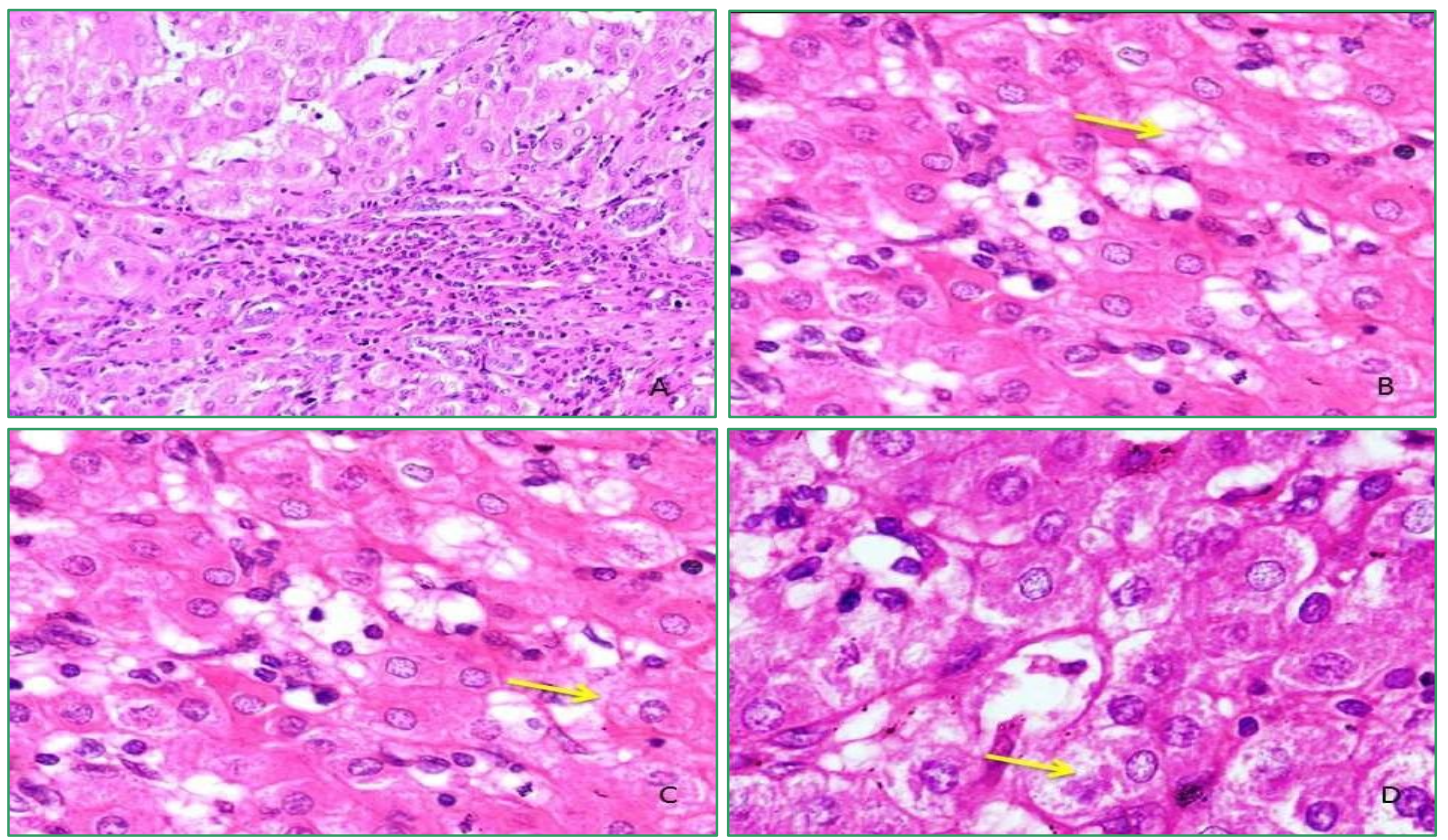

Figure (3) Different pathologic features of NAFLD; (A): portal inflammation, (B): focalmicrovesicular steatosis, (C): Ballooned hepatocytes, (D): Ballooned hepatocytes with intracytoplasmic inclusions "Mallory Denk Bodies" 


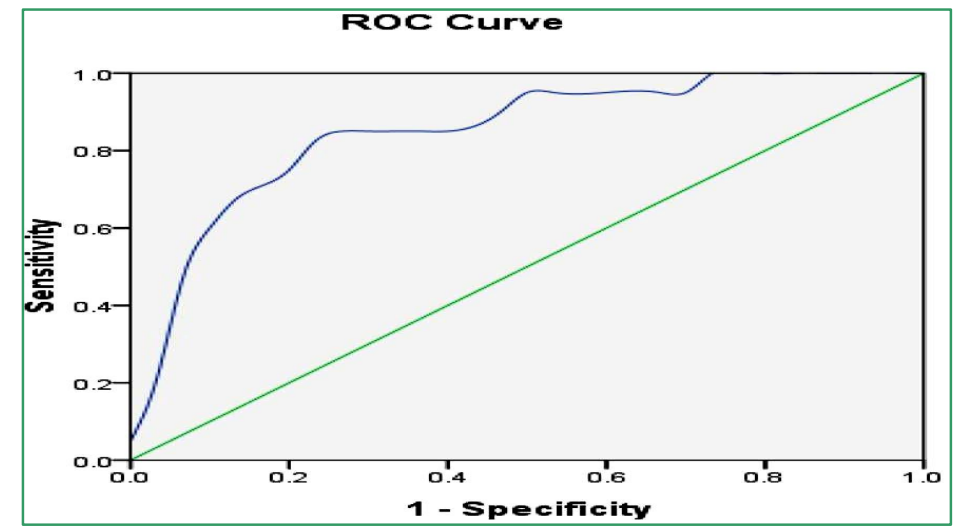

Figure (3) ROC curve showing the diagnostic sensitivity and specificity of CK-18 in diagnosing NASH

Table (3) The correlations between CK-18 and clinical and laboratory and histopathologic parameters of the NASH patients

\begin{tabular}{|lcc}
\hline \multicolumn{1}{c}{ The parameter } & R & P \\
\hline Age (years) & $\mathbf{0 . 0 5 1}$ & NS \\
BMI (kg/m2) & $\mathbf{0 . 7 1}$ & $<\mathbf{0 . 0 0 1}$ \\
\hline FBG (mg/dl) & $\mathbf{0 . 3 1 2}$ & $\mathbf{0 . 0 1 7}$ \\
MOMA-IR & $\mathbf{0 . 1 7 9}$ & $\mathbf{0 . 0 2 1}$ \\
AST (U/L) & $\mathbf{0 . 1 6}$ & NS \\
ALT (U/L) & $\mathbf{0 . 1 4}$ & NS \\
Gamma GT (U/L) & $\mathbf{0 . 1 8}$ & NS \\
\hline Total cholesterol (mg/dl) & $\mathbf{0 . 0 2 8}$ & NS \\
Triglycerides (mg/dl) & $\mathbf{0 . 0 8 3}$ & NS \\
\hline HDL (mg/dl) & $\mathbf{0 . 0 2 1}$ & NS \\
hc-CRP & $\mathbf{0 . 6 4}$ & $<\mathbf{0 . 0 0 1}$ \\
\hline Ferritin (ng/ml) & $\mathbf{0 . 0 4 2}$ & NS \\
\hline Steatosis & $\mathbf{0 . 4 2 1}$ & $<\mathbf{0 . 0 0 1}$ \\
\hline Fibrosis & $\mathbf{0 . 7 4}$ & $<\mathbf{0 . 0 0 1}$ \\
\hline Lobular inflammation & $\mathbf{0 . 5 6}$ & $<\mathbf{0 . 0 0 1}$ \\
\hline Ballooning & $\mathbf{0 . 3 9}$ & $<\mathbf{0 . 0 0 1}$ \\
\hline
\end{tabular}

\section{Discussion}

Non-alcoholic fatty liver disease (NAFLD) and its variant, non-alcoholic steatohepatitis (NASH) are steatotic liver diseases in the absence of significant alcohol intake. They are considered a hepatic sign of the metabolic syndrome, characterized by a spectrum of morphologic changes starting by macro vesicular steatosis, progressing to portal inflammation, ending up in liver cirrhosis. NAFLD is a common disease in the US and worldwide. Studies in the US found a significant association with obesity and diabetes, and it is even more common than alcoholic liver disease and hepatitis $\mathrm{C}$ with estimated 5 to 6 fold higher prevalence than that of chronic hepatitis $\mathrm{C}^{24}$. NASH is thought to eventually progress to liver cirrhosis, failure, and even carcinoma, unlike simple steatosis that is morphologically similar but harbors much better prognosis ${ }^{25}$. The diagnosis of NAFLD is based on clinical, radiologic, and morphologic characteristics. It combines the observation of persistently elevated serum aminotransferases with confirmatory imaging studies and supported by histopathological findings. The diagnosis is made in the setting of insignificant alcohol use, and any other chronic liver diseases. Liver biopsy is mandatory not only for confirming the diagnosis of NAFLD but for providing clues on the disease progression and grading of severity. It is also helpful in excluding the possibility of other chronic liver diseases. Despite being a major diagnostic tool for NAFLD, liver biopsy still has some limitations including the invasive nature of the procedure, being costly and requires hospitalization with the possibility of bleeding, the impact of biopsy size on the adequacy of biopsy for making the definitive diagnosis, and the pathologist experience role. The complexity of disease as captured in 
morphology and the similarity of alcoholic and NAFLD in terms of morphology are also limiting factors of liver biopsy ${ }^{13,26}$. Taking the above factors in consideration, there is a necessity to find non-invasive serological markers, efficiently considered as screening tests for NAFLD presence and to add to the prognostic criteria of NAFLD to identify patients likely to have NASH is mandatory ${ }^{27}$. A large body of evidence confirms the fundamental role of hepatocytes apoptosis in the pathogenesis of NAFLD and NASH ${ }^{11,28}$. CK18 is an intermediate filament protein that constitutes almost $5 \%$ of total liver proteins. It is considered a biomarker of hepatocellular apoptosis as it is cleaved, during apoptosis, at Asp 396 and recognized by M30 antibody specific for C-terminus ${ }^{29}$. A high prevalence of type $2 \mathrm{DM}$, dyslipidemia, obesity, and insulin resistance were noticed in our NAFLD and NASH patients compared to the control group. A result that was expected and supports other previous studies ${ }^{30-32}$ In NASH cases, significantly higher insulin resistance is found compared to cases with simple steatosis. This observation was similarly noted in different previous studies that declared the significance of insulin resistance in the pathogenesis and progress of $\mathrm{NASH}^{33,34}$. Our study showed significantly higher levels of aminotransferases (AST and ALT) in patients of simple steatosis and NASH compared to the healthy individuals. The relationship between elevation of aminotransferases and the progression of NASH to the fibrotic stage is somewhat complicated. Although elevated serum concentrations of ALT is known as a biomarker of liver injury and damage in NAFLD ${ }^{33,34}$, it does not necessarily indicate progression to the fibrotic stage. It was found that even NAFLD patients with normal ALT concentrations might still progress to NASH and advanced stage of fibrosis ${ }^{35}$. In the current study, significantly higher levels of AST were recorded in NASH patients in relation to the non-NASH group, a finding that was similarly supported by the study of Feldstein et $\mathrm{al}^{1}$. Accordingly, we can tell that patients with NAFLD associated with increased ALT levels are at increased risk to develop NASH along the disease course, but even in those patients with normal aminotransferases levels, NASH cannot be ruled out ${ }^{36}$. $\mathrm{Hc}-\mathrm{CRP}$ as an inflammatory factor was elevated in our NASH patients compared to the control group, supported by Park et al., who reported hc-CRP as an independent risk factor in nonalcoholic liver disease ${ }^{37}$. A significant elevation of serum level of ferritin in NASH patients was noted in our study, compared to both the control and the simple steatosis groups. A similar study conducted in Japan by Yoneda et al. supports the current result as it has stated that the serum level of ferritin was related to insulin resistance in NASH patients ${ }^{38}$. High levels of serum ferritin are not only simply explained by iron overload and liver accumulation but it could be also caused by hepatic inflammation as an inflammatory marker, oxidative stress and highly expressed cytokines ${ }^{39}$. There is strong evidence that serum CK-18M65 is released in NAFLD patients as a biomarker of apoptosis in NASH patients ${ }^{\mathbf{4 0 , 4 1}}$. The current study showed significantly higher serum levels of CK-18 in our patients with NAFLD and NASH compared to healthy group and in NASH compared to NAFLD group. Similarly, Wiekowska et al. has found that significantly higher levels of serum CK-18 are detected in NASH patients than NAFLD patients and healthy individuals, as an indicator of hepatic inflamemation $^{7}$. Essentially, CK-18 is found to be generated by caspase-3, which in turn is activated in the hepatocytes of NASH patients, and it is also found to be elevated in NASH compared to non-NASH patients ${ }^{28}$. The current results add to the growing body of evidence supporting the role of CK-18 in apoptosis induction and in NASH development. It is now unequivocally confirmed that CK-18 is an indicator of apoptosis and it is activated in NASH liver. Those findings along with our findings support the possibility of using CK-18 as a non-invasive marker for diagnosis and differentiation between $\mathrm{NAFL}$ and $\mathrm{NASH}^{42-45}$. A significantly positive association was discovered between serum CK-18 levels and all of fasting plasma glucose, body mass index, insulin resistance and $\mathrm{C}-$ reactive protein in NASH patients enrolled in the current study. This result was explained by Chitturri et al., who found a significant relationship between CK-18 and insulin resistance and confirmed its role in the pathogenesis and progression of NAFLD ${ }^{33}$. Similarly, Miyasato et al., observed a significant correlation between CK-18 and BMI in type 2 diabetic patients with $\mathrm{NASH}^{46}$. However, 
we failed to find significant correlations between serum CK-18 levels and aminotransferases, serum ferritin, the age of the patients and lipid profile in our NASH patients, the results suggested by Yilmaz et al., showed a weak correlation between serum CK-18 and the aminotransferases ${ }^{47}$. Serum CK-18 of our NASH patients correlates significantly with NAS scoring, the degree of steatosis, ballooning, lobular inflammation and fibrosis in our study. This is supported by Feldstein et al. and Wieckowska et $\mathrm{al}^{1,13}$. The results of Diab et al. and Vuppalanchi et al support our results about the association between serum levels of CK18 and NAS score in their patients ${ }^{48}$. Our study has limitations, like the small number of patients, uni-central study and absence of liver biopsy in the control group. So, a larger number multi-centric study can be done in the future.

\section{Conclusions}

In conclusion, serum CK-18 could be used as a non-invasive biomarker in NAFLD and NASH.

\section{List of Abbreviations}

CK -18: Cytokeratin-18, NAFL: Nonalcoholic fatty liver, NASH: nonalcoholic steatohepatitis, NAFLD: Nonalcoholic fatty liver disease, NAS: NAFLD Activity Score.

\section{References}

1- Feldstein E, Wieckowska A, Lopez R, Liu C, Zein N, McCullough J. Cytokeratin- 18 fragment levels as noninvasive biomarkers for nonalcoholic steatohepatitis: a multicenter validation study. Hepatology. 2009; 50 (4): 1072-1078.

2-Barsic N, Lerotic I, Smircic-Duvnjak L, Tomasic V, Duvnjak M. Overview and developments in noninvasive diagnosis of nonalcoholic fatty liver disease. World J. of Gastroenterology. 2012; 18 (30): 39453954.

3-Grigorescu M, Crisan D, Radu C, Grigorescu MD, Sparchez Z, Serban A. A novel pathophysiological-based panel of biomarkers for the diagnosis of nonalcoholic steatohepatitis. J. of Physiology and Pharmacology. 2012; 63 (4): 347-353.

4-Adams A, Angulo P. Treatment of non- alcoholic fatty liver disease. Postgraduate

Medical J. 2006; 82 (967): 315-322.

5- Petersen F, Dufour S, Hariri A, NelsonWilliams C, Foo N, Zhang M, et al. Apolipoprotein $\mathrm{C} 3$ gene variants in nonalcoholic fatty liver disease. The New England J. of Medicine. 2010; 362 (12): 1082-1089.

6- Farrell C, Larter Z. Nonalcoholic fatty liver disease: from steatosis to cirrhosis. Hepatology. 2006; 43 (2 Suppl 1): S99-S112.

7-Wieckowska A, McCullough J, Feldstein E. Noninvasive diagnosis and monitoring of nonalcoholic steatohepatitis: present and future. Hepatology. 2007; 46 (2): 582-589.

8-Ratziu V, Massard J, Charlotte F, Messous $\mathrm{D}$, Imbert-Bismut $\mathrm{F}$, Bonyhay L, et al. Diagnostic value of biochemical markers (FibroTest-FibroSURE) for the prediction of liver fibrosis in patients with nonalcoholic fatty liver disease. BMC Gastroenterology. 2006; 6:6.

9- Fealy E, Haus M, Solomon P, Pagadala M, Flask A, McCullough J, et al. Short-term exercise reduces markers of hepatocyte apoptosis in nonalcoholic fatty liver disease. J. Appl Physiol. 2012; 113: 1-6.

10- Guicciardi E, Gores J. Apoptosis: a mechanism of acute and chronic liver injury. Gut. 2005; 54 (7): 1024-1033.

11- Feldstein E, Gores J. Apoptosis in alcoholic and nonalcoholic steatohepatitis. Front Biosci. 2005; 10: 3093-3099.

12- Vos B, Barve S, Joshi-Barve S, Carew D, Whitington F, McClain J. Cytokeratin 18, a marker of cell death, is increased in children with suspected nonalcoholic fatty liver disease. J. of pediatric gastroenterology and nutrition 2008; 47(4): 481-485.

13- Wieckowska A, Zein N, Yerian M, Lopez $\mathrm{R}$, McCullough $\mathrm{J}$, Feldstein E. In vivo assessment of liver cell apoptosis as a novel biomarker of disease severity in nonalcoholic fatty liver disease. Hepatology. 2006; 44 (1): 27-33.

14- Younossi M, Jarrar M, Nugent $C$, Randhawa M, Afendy M, Stepanova M, et al. A novel diagnostic biomarker panel for obesity-related nonalcoholic steatohepatitis (NASH). Obesity Surgery. 2008; 18 (11): 1430-1437.

15- Diab L, Yerian L, Schauer P, Kashyap R, Lopez R, Hazen SL, et al. Cytokeratin 18 
fragment levels as a noninvasive biomarker for nonalcoholic steatohepatitis in bariatric surgery patients. Clinical Gastroenterology and Hepatology. 2008; 6(11):124954.

16- Yilmaz Y. Systematic review: caspasecleaved fragments of cytokeratin 18 - the promises and challenges of a biomarker for chronic liver disease. Alimentary Pharmacology \& therapeutics. 2009; 30 (1112): 1103-9.

17- Kleiner D, Brunt E, Van Natta $M$, Behling C, Contos M, Cummings O, et al. Design and validation of a histological scoring system for nonalcoholic fatty liver disease. Hepatology. 2005; 41 (6): 13131321.

18- Reitman S, Frankel S. A colorimetric met-hod for the determination of serum glutamic oxalacetic and glutamic pyruvic transom-inases. American J. of Clinical Pathology. 1957; 28 (1): 56-63.

19- Fiala S, Fiala AE, Dixon B. $\gamma$-Glutamyl Transpeptidase in Transplantable, Chemically Induced Rat Hepatomas and "Spontaneous" Mouse Hepatomas 2. J. of the National Cancer Institute. 1972; 48 (5): 1393- 13401.

20- King J, Armstrong R. Practical Clinical Biochemistry. Varley eH, editor: CBS Publishers, New Delhi: CBS 1988: 458.

21- Stiehl A. Hyperbilirubinemia in liver dise-ases. Fortschr Med. 1982; 100 (18): 842-845.

22- Friedewald T, Levy I, Fredrickson S. Estimation of the concentration of lowdensity lipoprotein cholesterol in plasma, without use of the preparative ultracentrifuge. Clinical Chemistry. 1972; 18 (6): 499-502.

23- Müller W, Mierau R, Wohltmann D. Interference of $\operatorname{IgM}$ rheumatoid factor with nephelometric C-reactive protein determinations. J. of Immunological Methods. 1985; 80 (1): 77-90.

24- Williams D, Stengel J, Asike I, Torres M, Shaw J, Contreras M, et al. Prevalence of nonalcoholic fatty liver disease and nonal-coholic steatohepatitis among a largely middle-aged population utilizing ultra-sound and liver biopsy: a prospective study. Gastroenterology. 2011; 140 (1):
124-131.

25- Chalasani N, Younossi Z, Lavine E, Diehl M, Brunt M, Cusi K, et al. The diagnosis and management of nonalcoholic fatty liver disease: practice Guideline by the American Association for the Study of Liver Diseases, American College of Gastroenterology, and the American Gastroenterological Association. Hepatology. 2012; 55 (6): 2005- 2023.

26- Nalbantoglu L, Brunt M. Role of liver biopsy in nonalcoholic fatty liver disease. World J of Gastroenterology. 2014; 20 (27): 9026-9037.

27- Poynard T, Morra R, Ingiliz P, ImbertBismut F, Thabut D, Messous D, et al. Assessment of Liver Fibrosis: Noninvasive Means. Saudi J. of Gastroenterology. 2008; 14 (4): 163-173.

28- Feldstein E, Canbay A, Angulo P, Taniai M, Burgart J, Lindor D, et al. Hepatocyte apoptosis and fas expression are prominent features of human nonalcoholic steatohep-atitis. Gastroenterology. 2003; 125 (2): 437-443.

29- Leers $P$, Kolgen $W$, Bjorklund V, Bergman T, Tribbick G, Persson B, et al. Immunocytochemical detection and mapping of a cytokeratin 18 neo-epitope exposed during early apoptosis. The J. of Pathology. 1999; 187 (5): 567-572.

30- Malik R, Chang M, Bhaskar K, Nasser I, Curry M, Schuppan D, et al. The clinical utility of biomarkers and the nonalcoholic steatohepatitis CRN liver biopsy scoring system in patients with nonalcoholic fatty liver disease. J. of Gastroenterology and Hepatology. 2009; 24 (4): 564-568.

31- Miele L, Forgione A, La Torre G, Vero V, Cefalo C, Racco S, et al. Serum levels of hyaluronic acid and tissue metalloproteinase inhibitor-1 combined with age predict the presence of nonalcoholic steatohepatitis in a pilot cohort of subjects with nonalcoholic fatty liver disease. Translational Research. 2009; 154 (4): 194-201.

32- Tsutsui M, Tanaka N, Kawakubo M, Sheena Y, Horiuchi A, Komatsu M, et al. Serum fragmented cytokeratin 18 levels reflect the histologic activity score of non-alcoholic fatty liver disease more 
accurately than serum alanine aminotransferase levels. J. of Clinical Gastroenterology. 2010; 44 (6): 440-447.

33- Chitturi S, Abeygunasekera S, Farrell GC, Holmes-Walker J, Hui JM, Fung C, et al. NASH and insulin resistance: Insulin hypersecretion and specific association with the insulin resistance syndrome. Hepatology. 2002; 35 (2): 373-379.

34- Hussien H, Pasha H, Mostafa N. Apoptotic Shards of Cytokeratin 18 Point to Steatohepatitis in patients with non alcoholic hepatic steatosis. Med J. Cairo Univ. 2011; 79 (2): 199-205.

35- Mofrad P, Contos J, Haque M, Sargeant C, Fisher A, Luketic A, et al. Clinical and histologic spectrum of nonalcoholic fatty liver disease associated with normal ALT values. Hepatology. 2003; 37 (6): 12861292.

36- Adams A, Feldstein E. Non-invasive diagnosis of nonalcoholic fatty liver and nonalcoholic steatohepatitis. J. of Digestive Diseases. 2011; 12(1): 10-6.

37- Park H, Kim I, Yun W, Kim W, Park I, Cho YK, et al. Insulin resistance and Creactive protein as independent risk factors for non-alcoholic fatty liver disease in non- obese Asian men. J. of Gastroenterology and Hepatology. 2004; 19 (6): 694-698.

38- Yoneda M, Nozaki Y, Endo H, Mawatari $\mathrm{H}$, Iida $\mathrm{H}$, Fujita K, et al. Serum ferritin is a clinical biomarker in Japanese patients with nonalcoholic steatohepatitis (NASH) independent of HFE gene mutation. Digestive Diseases \& Sciences. 2010; 55 (3): 808-814.

39- Sumida Y, Eguchi Y, Ono M. Current status and agenda in the diagnosis of nonalcoholic steatohepatitis in Japan. World J. of Hepatology. 2010; 2 (10): 374-383.

40- Haukeland W, Damas K, Konopski Z, Loberg M, Haaland T, Goverud I, et al. Systemic inflammation in nonalcoholic fatty liver disease is characterized by elevated levels of CCL2. J. of Hepatology. 2006; 44 (6): 1167-1174.

41- Shimada M, Kawahara H, Ozaki K,
Fukura M, Yano H, Tsuchishima M, et al. Usefulness of a combined evaluation of the serum adiponectin level, HOMA-IR, and serum type IV collagen $7 \mathrm{~S}$ level to predict the early stage of nonalcoholic steatohepatitis. The American J. of Gastroenterology. 2007; 102 (9): 1931-1938.

42- Miller H, Ferguson A, Dillon F. Systematic review of performance of non- invasive biomarkers in the evaluation of nonalcoholic fatty liver disease. Liver Int. 2011; 31 (4): 461-473.

43- Shen J, Chan L, Wong L, Choi C, Chan A, Chan Y, et al. Non-invasive diagnosis of non-alcoholic steatohepatitis by combined serum biomarkers. J. of Hepatology. 2012; 56 (6): 1363-1370.

44- Tamimi I, Elgouhari M, Alkhouri N, Yerian M, Berk P, Lopez R, et al. An apoptosis panel for nonalcoholic steatohepatitis diagnosis. J. of Hepatology. 2011; 54 (6): 12241229.

45- Younossi M, Page S, Rafiq N, Birerdinc A, Stepanova M, Hossain N, et al. A biomarker panel for non-alcoholic steatohepatitis (NA $\mathrm{SH})$ and NASH-related fibrosis. Obesity Surgery. 2011; 21 (4): 431-439.

46- Miyasato M, Murase-Mishiba Y, Bessho M, Miyawaki M, Imbe $\mathrm{H}$, Tsutsumi C, et al. The cytokeratin-18 fragment level as a bio-marker of nonalcoholic fatty liver disease in patients with type 2 diabetes mellitus. Clinica Chimica Acta. 2014; 433: 184-189.

47- Yilmaz Y, Dolar E, Ulukaya E, Akgoz S, Keskin M, Kiyici M, et al. Soluble forms of extracellular cytokeratin 18 may differentiate simple steatosis from nonalcoholic steatohepatitis. World J. of Gastroenterology 2007; 13 (6): 837-844.

48- Vuppalanchi R, Deppe RB, Yates KP, Comerford M, Masuoka HC, Tetri BA, et al. Changes in serum cytokeratin 18 levels significantly predict changes in liver histology in adults with nonalcoholic steatohepatitis: Results from the pivens trial. Gastroenterology 2013; 144 (5): S-95 